Pantoja-Vallejo, A., Colmenero Ruiz, M.J. y Molero, D. (2022). Aspectos condicionantes de la tutoría universitaria. Un estudio comparado. Revista de Investigación Educativa, 40(1), 33-49.

DOI: http://dx.doi.org/10.6018/rie.373741

\title{
Aspectos condicionantes de la tutoría universitaria. Un estudio comparado
}

\section{Determinant aspects of university tutoring. A comparative study}

\author{
Antonio Pantoja-Vallejo, María Jesús Colmenero Ruiz, David Molero' \\ Departamento de Pedagogía, Universidad de Jaén (España)
}

\begin{abstract}
Resumen
Las universidades de todo el mundo están poniendo en marcha diferentes acciones de orientación y acompañamiento al alumnado. Para analizar este fenómeno, se plantea un estudio en una universidad española y en otra argentina. Se diseña y valida una escala tipo Likert tomando como variables el género del alumnado, la universidad de estudio y la participación o no en el Plan de Acción Tutorial (PAT). Intervienen un total de 866 estudiantes (314 hombres y 552 mujeres). Los resultados indican una buena valoración de los aspectos relacionados con el PAT y la tutoría, no se perciben diferencias significativas entre alumnos y alumnas, pero sí se aprecian entre ambas universidades, a favor de la universidad argentina en la estimación positiva del PAT y de la tutoría, mientras que esta tendencia se invierte en el uso de las TIC. En ambos casos, se admite que la participación en el PAT mejora la tutoría.

Palabras clave: tutoría; orientación; universidad; tecnología educativa.
\end{abstract}

\begin{abstract}
Correspondencia: Antonio Pantoja-Vallejo, apantoja@ujaen.es. Universidad de Jaén (España). Facultad de Humanidades y CCEE. Departamento de Pedagogía Área de Métodos de Investigación y Diagnóstico en Educación. (MIDE). Campus Las Lagunillas, s/n. Edificio C5. Despacho 236. 23071 Jaén.

1 Este estudio forma parte del proyecto de investigación "Desarrollo de estrategias de orientación y tutoría en profesorado universitario europeo y latinoamericano mediante el uso de Tecnologías de la Información y la Comunicación (TIC)" (UJA2016/08/12), subvencionado con fondos de la Universidad de Jaén (España).
\end{abstract}


Universities around the world are launching different guidance and support programs for undergraduate students. To analyze this phenomenon, we propose a comprehensive study of the tutoring methods of a Spanish and an Argentinian university. A Likert-type scale has been designed and validated for this purpose, taking into consideration the students' gender, the university of study, and the participation (or lack thereof) in the Tutorial Action Plan (TAP). A total of 866 students (314 men and 552 women) have been involved in this study. The results indicate a good assessment of the aspects related to the TAP and the tutoring service and show no significant differences in the male and female students' perceptions of the programs. There is a notable distinction, however, in the results obtained by each university, noting a very positive assessment of the Argentinian university's TAP. Interestingly, this trend was reversed when assessing the use of ICT in both institutions. The study concludes that participation in the TAP makes tutoring more effective.

Keywords: tutoring; guidance; higher education; educational technology

\section{Introducción}

La orientación y la tutoría en la Educación Superior es uno de los tópicos que, a nivel teórico, más se trabajan tanto en el escenario nacional como internacional en estos últimos años. Su protagonismo (Muñoz y Gairín, 2014), no sólo reside en el importante papel que tiene en relación con la formación integral de los estudiantes, sino en el rol que juega al ser uno de los ejes articuladores del enfoque educativo centrado en el alumnado y el aprendizaje. A nivel práctico, es una cuestión en la que todavía no se ha profundizado suficientemente, entre otras razones, porque en la función docente del profesorado se priorizan otras acciones y por la falsa creencia de que estos estudiantes son ya personas adultas, autónomas y maduras para gestionar su proyecto formativo, con escasa necesidad de asesoramiento; apreciación ésta, bastante alejada de la realidad.

Además del impulso dado a la tutoría por el Espacio Europeo de Educación Superior (EEES), en España se han publicado leyes y decretos específicos, pero en especial es el Estatuto del Estudiante (RD 1791/2010), el que da un impulso significativo a la orientación y tutoría como derecho de los estudiantes en los tres ciclos de las enseñanzas universitarias. En este contexto, se señala que el profesorado universitario, haya sido designado o no como tutor de un grupo de estudiantes (Brical, 2000), tiene que ofrecer un número de horas de tutorías a la semana en función de su dedicación. Sin embargo, a pesar de ello, se observa cómo, en la Educación Superior, a la figura del tutor no se le presta suficiente atención, dejando de tener en cuenta los beneficios que pueden aportar al alumnado (Colvin y Ashman, 2010; Fox, Stevenson, y Connelly, 2010; Watts, 2011).

En referencia a Argentina, tomada como referencia en el estudio comparativo, no existe un modelo claro de implantación de las tutorías en las universidades, si bien en el caso específico de la Universidad Agustín Maza de Mendoza (Argentina), se viene implementando un sistema de tutorías desde el año 1997. Se organiza para dar respuesta a las necesidades vocacionales, académicas y personales que presentan los estudiantes durante el primer año de su formación universitaria, de forma que le pueda ser útil en el proceso de inserción en la universidad.

Parece claro, pues, el esfuerzo legislativo y de implementación en las universidades por parte del profesorado, en una consideración novedosa de la figura del tutor y de 
los planes de acción tutorial (Muñoz y Gairín, 2014). Sin embargo, es conveniente la necesidad de desarrollar acciones de orientación y acompañamiento previas al ingreso a la universidad, tales como los planes de tutoría, mediante los que se puede lograr una mejora en los procesos de transición y adaptación del alumnado a la universidad, actividades propedéuticas para detectar y corregir lagunas en la formación previa y actuaciones de acogida para estudiantes de nuevo ingreso (Álvarez, 2012; Cabrera, Bethencourt, Álvarez, y González, 2006; Donoso, 2010; Gallego, 2004; Lim, 2002; Tinto, 1975). Es preciso diseñar situaciones significativas de aprendizaje en las que se trabajen aspectos relacionados con la orientación personal, académica y profesional de sus alumnos (De Backer et al., 2015; Hagenauer y Volet, 2014; López, 2017).

En este sentido, se constata la relevancia de la función tutorial en la multiplicidad de estudios sobre esta temática, según sea la modalidad en la que se desarrolla, vocacional, académica o profesional (Cano, 2009; Capelari, 2014); aportaciones que ofrece al alumnado un mayor seguimiento académico y una orientación profesional (Lorenzo-Lledó, Lledó, Lorenzo, y Arráez, 2017; Solaguren y Moreno, 2019); y más específicamente lo que puede contribuir a la formación integral de los estudiantes y a una educación de calidad (Álvarez, 2013; Fernández, Arco, López y Heilborn, 2011; Gaughf y Foster, 2016; Larose et al. 2011), en los instrumentos utilizados para determinar su calidad (Caldera et al., 2015), o en su utilidad desde el punto de vista del alumnado (Álvarez, 2013; De la Cruz, 2017) o del profesorado, y en el uso de las TIC para su desarrollo (Pantoja, 2009).

Los estudiantes, por su parte, consideran que la tutoría sirve fundamentalmente para orientar la asignatura y su evaluación. No están lo suficientemente concienciados de la importancia de las tutorías más allá del asesoramiento técnico ceñido a las materias, limitándolas a la resolución de dudas de clase (García y Troyano, 2009; Martínez y Crespo, 2009; Giménez, Cortés y Motos, 2018). Por estas razones, se considera necesario fomentar la participación de los estudiantes en las actividades de acción tutorial y potenciar las entrevistas con los tutores asignados (Aguilar-Parra et al., 2015; De-Juan-Vigaray y García, 2017; Santos et al., 2012), del mismo modo que establecer un reconocimiento del sistema de créditos que ayudará a mejorar la calidad del conocimiento transferible y la valiosa tutoría de los estudiantes en la educación superior (Arrebola et al., 2016). Existen estudios que avalan los beneficios de los planes de acción tutoriales en los alumnos universitarios en el sentido de que experimentan habilidades relacionadas con la comunicación, utilizando regularmente los conocimientos académicos que habían adquirido (Hall y Jaugietis, 2011; McKeegen, 1998; Ocel et al., 2003; Schalk et al., 2009), en el sentimiento de pertenencia que adquieren hacia la universidad (Strayhorn, 2012), en el desarrollo de contenidos académicos, docentes, personales y profesionales (AlonsoGarcía, Rodríguez y Cáceres, 2018; Da Re, Clerici y Álvarez, 2017).

Con respecto al uso que se hace de las tecnologías de la información y la comunicación (TIC) en la acción tutorial, encontramos posiciones encontradas ya que para algunos autores (De-Juan-Vigaray y García, 2017), el correo electrónico es una herramienta de comunicación imprescindible en estos momentos, un recurso que facilita la interacción profesor-alumnos, ya que agiliza el contacto de una forma más flexible, frente a otros (Alonso-García, Rodríguez y Cáceres, 2018) que se sienten disconformes con la acción tutorial universitaria como acción de utilidad para promover el uso de 
las TIC en el desarrollo de la tutoría (videollamadas, chats, redes sociales, etc.). Del mismo modo, el profesorado que utiliza foros de dudas y comentarios en plataformas virtuales destaca la gran utilidad de este recurso (García, 2008).

No obstante, para algunos autores (Cabello y Antón, 2005; Marqués, 2001), resulta evidente la conveniencia de complementar las tutorías presenciales con la posibilidad de contacto telemático (sea correo electrónico, chat o videoconferencia a través de Internet). En líneas generales, el profesorado utiliza las TIC, pero es evidente también la necesidad de orientar estas prácticas educativas hacia un uso más adecuado y productivo desde el punto didáctico (Martínez y González, 2015).

El objetivo principal de este estudio es analizar la acción tutorial desde la perspectiva de los estudiantes en dos universidades diferentes, una europea (Universidad de Jaén, España) y otra americana (Universidad Agustín Maza, Argentina), con especial atención a los Planes de Acción Tutorial (PAT). Se plantea como hipótesis que el contexto de la universidad condiciona la tutoría universitaria y que el género, junto con la participación en el PAT, influyen en la satisfacción de los estudiantes en los estudios cursados.

\section{Método}

Se sigue un diseño no experimental de tipo descriptivo-correlacional con la finalidad de comparar los datos más significativos de ambas universidades participantes en el estudio, realizándose medidas de carácter transversal.

\section{Participantes}

La población está compuesta por el alumnado participante en los PAT en ambas universidades, siendo este un número cambiante a lo largo del curso académico. La muestra la forman 866 sujetos (Tabla 1), de los cuales 552 (63.7\%) son mujeres y 314 (36.3\%) hombres.

No se realizó un proceso de selección muestral, ya que se buscaba la participación de aquellos alumnos de primer año que cursaran estudios con PAT en funcionamiento, por lo que se empleó un muestreo no probabilístico intencional. En la Tabla 1 se refleja la distribución por género y universidad.

Tabla 1

Distribución de los participantes por género y universidad

\begin{tabular}{lccc}
\hline Género & UJAEN & UMAZA & Total \\
\hline Mujeres & $206(23.8 \%)$ & $346(40.4 \%)$ & $552(63.7 \%)$ \\
Hombres & $118(13.6 \%)$ & $196(22.6 \%)$ & $314(36.3 \%)$ \\
Total & $324(37.4 \%)$ & $542(62.6 \%)$ & $866(100 \%)$ \\
\hline
\end{tabular}




\section{Instrumento}

Para la recogida de datos, se ha utilizado una escala tipo Likert diseñada ad hoc, de cuatro opciones de respuesta, que van de 1 (en completo desacuerdo) a 4 (totalmente de acuerdo), antecedida de 9 preguntas de identificación. En su construcción se siguieron tres fases: a) revisión bibliográfica de aquellos factores relacionados con el desarrollo de las tutorías en la universidad y cuestiones relevantes para el estudio; b) selección de un grupo de 6 tutores con experiencia de un mínimo de 5 años (validez de contenido), que expusieron su juicio crítico sobre los elementos iniciales de la escala; c) validación de constructo del instrumento con una muestra de 280 alumnos. El instrumento final, "La tutoría universitaria y el Plan de Acción Tutorial (PAT). Cuestionario para alumnado", quedó constituido por 32 ítems divididos en tres factores y se aplicó de forma auto-administrada, en unos casos de forma presencial y otros a través de internet (Díaz, 2012; Torrado, 2004) mediante el correspondiente formulario de Google Drive.

Con respecto a la validez de constructo, ésta se obtiene mediante un análisis factorial exploratorio por el método de los componentes principales como forma de agrupar los ítems en dimensiones que puedan dar una explicación de la varianza observada en las respuestas de los sujetos. El índice de adecuación muestral, KMO, alcanza un valor de .959 y la prueba de esfericidad de Bartlett es de $13060.178(p<.000)$. Una vez rechazada la hipótesis nula de que la matriz de correlación inter-ítems es identidad al comprobar que las respuestas están sustancialmente relacionadas, se procede al análisis de los componentes principales y se comprueban tres iteraciones que explican un total del $53.41 \%$ de la varianza. Al primer componente denominado Plan de Acción Tutorial (PAT), le corresponde un $23.73 \%$ de la varianza, al segundo llamado Situación actual de la Tutoría (SIT), un 21.63\% y al tercero denominado Tecnologías de la Información y Comunicación (TIC) un $7.83 \%$.

Finalmente, la consistencia interna del instrumento ha sido calculada mediante el coeficiente Alfa de Cronbach, siendo la fiabilidad de las puntaciones para el total de la escala de .93 y para cada una de las dimensiones que componen el cuestionario de .80 (PAT), .93 (SIT) y .68 para la dimensión TIC, siendo las correlaciones entre las puntuaciones de las tres dimensiones significativas en todos los casos (véase Tabla 2).

Tabla 2

Correlaciones ( $r$ ) entre los factores, descriptivos y fiabilidad de las puntuaciones

\begin{tabular}{lccc}
\hline Género & PAT [Rango 6-24] & SIT [Rango 16-64] & TIC [Rango 10-40] \\
\hline PAT & - & & \\
SIT & $.74^{* *}$ & - & - \\
TIC & $.42^{* *}$ & $.51^{* *}$ & $19.13( \pm 5.15)$ \\
M $(D T)$ & $16.54( \pm 4.54)$ & $43.31( \pm 12.38)$ & .68 \\
Alpha $(\alpha)$ & .80 & .93 & \\
\hline
\end{tabular}

Nota: (1) PAT=Plan de Acción Tutorial, SIT=Situación Actual de la Tutoría, TIC=Tecnologías Información y la Comunicaci global $\odot$ Valoracincia Emocionalón. (2) ${ }^{*}=p<.05 ;{ }^{* *}=p<.01$. (3) $M=$ Media, $D T=$ Desviación Típica, $\alpha=$ Fiabilidad de las puntuaciones (consistencia interna). 


\section{Procedimiento de recogida y análisis de datos}

La administración del cuestionario fue posible gracias a la colaboración de las autoridades académicas de ambas universidades, que facilitaron los datos para conocer el número de PAT existentes, el alumnado y tutores involucrados. Se contactó con los coordinadores de los PAT y con los propios tutores, informando de la investigación y de las dos modalidades de participación: a) en las mismas clases, previa autorización del tutor y con consentimiento del alumnado; b) en Internet, cumplimentando la escala disponible en Google Drive.

Para el análisis de los datos se empleó IBM SPSS Statistics versión 22 (SPSS Inc., Chicago, USA). Se ha realizado un análisis factorial exploratorio con rotación Varimax con Kaiser, un estudio descriptivo (porcentajes, medias, desviaciones típicas), y para conocer la existencia de diferencias significativas en función de las variables sociodemográficas (género, universidad y participación en el PAT) se utilizó la prueba $t$ de Student de diferencia de medias para muestras independientes. Se han verificado los supuestos de independencia de los datos, normalidad y homocedasticidad y se informa del tamaño del efecto, empleándose pruebas paramétricas al sostenerse la asunción de normalidad en todos los casos. Para finalizar, se realiza un estudio de regresión lineal mediante el método de pasos sucesivos para predecir qué dimensiones explican en mayor medida la tutoría en la universidad. En los análisis se ha empleado un nivel de confianza del 95\% (significación $p<.05$ ).

\section{Resultados}

\section{Valoración de la tutoría universitaria}

Para dar respuesta al primero de los objetivos, una vez que se ha presentado el proceso de elaboración y validación del instrumento, se presentan los resultados obtenidos para el total de la muestra $(n=866)$ en cada uno de los factores del cuestionario y la correlación existente entre las puntuaciones de los mismos (véase Tabla 2). Las correlaciones entre los factores son positivas y significativas en todos los casos y de magnitud moderada o alta (valores $r_{886}$ comprendidos entre .42 y .74; $p<.01$ ). Las valoraciones de cada factor son elevadas, superando claramente de manera global las puntuaciones intermedias en cada dimensión en función del rango de puntuaciones posibles.

Tabla 3

Estadísticos descriptivos de las preguntas más y menos valoradas de cada factor

$\operatorname{Media}(M)$ D. Típica

Más valoradas del Plan de Acción Tutorial (PAT)

PAT.4. El número de estudiantes por tutor asignado me parece $3.01 \quad \pm 1.04$ adecuado.

PAT.1. Me han informado de los objetivos del PAT.

$2.96 \pm 1.06$




\begin{tabular}{|c|c|c|}
\hline & $\operatorname{Media}(M)$ & D. Típica \\
\hline $\begin{array}{l}\text { PAT.6. Las tutorías se deben mantener durante todo el periodo de mi } \\
\text { estancia en la universidad. }\end{array}$ & 2.96 & \pm 1.10 \\
\hline \multicolumn{3}{|l|}{ Menos valorada del Plan de Acción Tutorial (PAT) } \\
\hline $\begin{array}{l}\text { PAT.2. He participado en reuniones o debates sobre tutoría en la } \\
\text { Universidad. }\end{array}$ & 2.23 & \pm 1.10 \\
\hline Promedio Factor Plan de Acción Tutorial (PAT) & 2.76 & \pm .75 \\
\hline \multicolumn{3}{|l|}{ Más valoradas Situación Actual de la Tutoría (SIT) } \\
\hline SIT.1. La tutoría en la universidad la valoro positivamente. & 3.12 & \pm .94 \\
\hline $\begin{array}{l}\text { SIT.5. La tutoría proporciona una orientación durante mi estancia en la } \\
\text { Universidad en itinerarios, optatividad, etc. }\end{array}$ & 2.89 & \pm 1.00 \\
\hline \multicolumn{3}{|l|}{ Menos valorada Situación Actual de la Tutoría (SIT) } \\
\hline $\begin{array}{l}\text { SIT.7. La tutoría proporciona una orientación en movilidad nacional e } \\
\text { internacional. }\end{array}$ & 2.35 & \pm 1.02 \\
\hline Promedio Factor Situación Actual de la Tutoría (SIT) & 2.71 & \pm .77 \\
\hline \multicolumn{3}{|l|}{ Más valoradas de TIC } \\
\hline $\begin{array}{l}\text { TIC.10. Se utilizan las TIC para la coordinación entre los compañeros } \\
\text { tutelados. }\end{array}$ & 3.14 & \pm 1.27 \\
\hline $\begin{array}{l}\text { TIC.6. Utilizo un grupo de Whatsapp para estar en contacto con el tutor } \\
\text { y otros compañeros tutelados. }\end{array}$ & 3.03 & \pm 1.37 \\
\hline \multicolumn{3}{|l|}{ Menos valoradas de TIC } \\
\hline $\begin{array}{l}\text { TIC.7. En la Web de la Universidad utilizo un foro específico sobre } \\
\text { tutoría. }\end{array}$ & 1.14 & \pm .549 \\
\hline Promedio Factor TIC & 1.91 & \pm .514 \\
\hline
\end{tabular}

Nota: (1) PAT=Plan de Acción Tutorial, SIT=Situación Actual Tutoría, TIC=Tecnologías Información y la Comunicaci global ๑) Valoracincia Emocionalón.

Asimismo, se presenta un estudio descriptivo de las variables más y menos valoradas de cada uno de los tres factores del instrumento, para analizar qué aspectos son los mejor y peor considerados (véase Tabla 3). Tal y como se aprecia, los dos primeros factores son los que de manera global son los mejor valorados por los sujetos de la muestra, el Plan de Acción Tutorial en primer lugar $(M=2.76 \pm .75)$, seguido de la Situación Actual de la Tutoría con una valoración similar $(M=2.71 \pm .77)$ y, por último, el factor Tecnologías de la Información y Comunicación, el único que presenta una valoración media inferior a 2 puntos de manera global $(M=1.91 \pm .51)$.

Las valoraciones medias más altas del primer factor (PAT) están relacionadas con el número de estudiantes por tutor (PAT.4), los objetivos (PAT.1) y la duración del PAT (PAT.6), siendo la cuestión menos valorada la relacionada con la participación de los estudiantes en reuniones relacionadas con el Plan de Acción Tutorial (PAT.2). En el factor Situación Actual de la Tutoría (SIT), las valoraciones más altas están en la valoración de la tutoría (SIT.5) y en la orientación recibida sobre itinerarios y optatividad (SIT.5), siendo lo menos considerado la información facilitada sobre la movilidad nacional e internacional (SIT.7). En el tercer factor (TIC), a pesar de obtenerse la menor valoración media de manera global, aparecen cuestiones con medias altas $(M>3.0)$ como el empleo de las TIC para 
contactar con los estudiantes tutelados (TIC.10) y el empleo de servicios de mensajería instantánea como medio de contacto entre tutores y estudiantes (TIC.6), aunque aparecen algunas cuestiones con reducidas puntuaciones medias relacionadas con el empleo o uso de algunos recursos TIC para el desarrollo de la tutoría universitaria (TIC.7).

\section{Diferencias en función de las variables sociodemográficas}

Para analizar la existencia de diferencias estadísticamente significativas entre cada una de las variables de identificación consideradas: género (hombre vs. mujer), universidad (UJA vs. UMAZA) y participación en el PAT (sí vs. no), tal y como contempla el segundo objetivo de nuestro estudio, se han realizado distintas pruebas $t$ de Student de diferencias de medias para muestras independientes, tal y como aparece en la Tabla 4. En relación al género, no se han encontrado diferencias significativas en ningún caso $(p>.05)$, estando las puntuaciones medias situadas en valores muy próximos entre los hombres y las mujeres, de manera global.

Por el contrario, en la variable universidad (UJA-España- vs. UMAZA-Argentina-) sí hay diferencias significativas en los tres factores. En el PAT $\left(t_{866}=8.55, p=.00, d=-.62\right)$ la diferencia es favorable a la UMAZA, siendo el valor del tamaño del efecto, mediano ( $d=-.62)$ según los criterios clásicos de Cohen (1988). En la SIT $\left(t_{866}=9.78, p=.00, d=-.71\right)$ la significatividad también es a favor de los estudiantes de la UMAZA de manera destacada, estando el tamaño del efecto cercano a valores altos $(d=-.71)$. En el factor TIC, son los estudiantes de la Universidad de Jaén (UJA) quienes puntúan más alto $\left(t_{866}=8.94, p=.00, d=.68\right)$ siendo el tamaño del efecto mediano.

Por último, en relación a la participación en el PAT de las universidades (Sí vs. No), se obtienen diferencias en los tres factores (PAT, SIT y TIC) a favor de los estudiantes que si han participado en las iniciativas de sus universidades para el desarrollo de la tutoría. En todos los casos, las diferencias son significativas de manera clara a favor de los que sí han experimentado la oportunidad de participar en el PAT $(p<.01)$ con respecto a los que no lo han hecho. Es preciso destacar los resultados obtenidos en los factores PAT $\left(t_{866}=15.63, p=.00, d=1.15\right)$ y $\operatorname{SIT}\left(t_{866}=12.87, p=.00, d=.96\right)$, no solo por los elevados valores obtenidos en el estadístico $t$ de Student, sino también por los valores del tamaño del efecto ( $d=1.15$ y $d=.96$, respectivamente) siendo ambos calificados como grandes o elevados (Cohen, 1988), cuestión que justifica que la significatividad en las diferencias de las puntuaciones se debe a la influencia de la participación o no en las iniciativas universitarias de tutoría destinadas a los estudiantes.

Tabla 4

Diferencias de medias en función del género ( $t$ de Student)

\begin{tabular}{cccccc}
\hline Género & Hombres $(\mathrm{n}=\mathbf{3 1 4}) \boldsymbol{M}(\mathbf{D T})$ & $\begin{array}{c}\text { Mujeres }(\mathrm{n}=552) M \\
(\mathrm{DT})\end{array}$ & $\boldsymbol{t}(\mathbf{8 6 4})$ & $\boldsymbol{p}$ & $\begin{array}{c}\text { Efecto } \\
(\boldsymbol{d})\end{array}$ \\
\hline PAT (Rango 6-24) & $16.65( \pm 4.36)$ & $16.47( \pm 4.64)$ & -.55 & .58 & .03 \\
SIT (Rango 16-64) & $44.01( \pm 11.26)$ & $42.92( \pm 12.96)$ & -1.23 & .21 & .08 \\
\hline
\end{tabular}




\begin{tabular}{|c|c|c|c|c|c|}
\hline Género & Hombres $(\mathrm{n}=314) M(D T)$ & $\begin{array}{c}\text { Mujeres }(\mathrm{n}=552) M \\
(D T)\end{array}$ & $t(864)$ & $p$ & $\begin{array}{l}\text { Efecto } \\
\text { (d) }\end{array}$ \\
\hline TIC (Rango 10-40) & $19.08( \pm 5.26)$ & $19.22( \pm 4.96)$ & -.40 & .68 & -.02 \\
\hline Universidad & $\begin{array}{c}\text { UJA }(n=324) \\
M(D T)\end{array}$ & $\begin{array}{c}\text { UMAZA }(n=542) \\
M(D T)\end{array}$ & $t_{(864)}$ & $p$ & Efecto $(d)$ \\
\hline PAT (Rango 6-24) & $14.78( \pm 5.11)$ & $17.59( \pm 3.80)$ & 8.55 & $.00^{* *}$ & -.62 \\
\hline SIT (Rango 16-64) & $37.85( \pm 14.13)$ & $46.58( \pm 9.86)$ & 9.78 & $.00^{* *}$ & -.71 \\
\hline TIC (Rango 10-40) & $20.51( \pm 2.65)$ & $16.83( \pm 7.12)$ & 8.94 & $.00^{* *}$ & .68 \\
\hline Participación PAT & $\begin{array}{l}\text { Si PAT }(n=553) \\
\quad M(D T)\end{array}$ & $\begin{array}{c}\text { No PAT }(n=300) \\
M(D T)\end{array}$ & $t_{(851)}$ & $p$ & Efecto $(d)$ \\
\hline PAT (Rango 6-24) & $18.22( \pm 3.56)$ & $13.47( \pm 4.57)$ & 15.63 & $.00^{* *}$ & 1.15 \\
\hline SIT (Rango 16-64) & $47.23( \pm 9.72)$ & $35.89( \pm 13.47)$ & 12.87 & $.00^{* *}$ & .96 \\
\hline TIC (Rango 10-40) & $20.16( \pm 3.94)$ & $17.13( \pm 6.43)$ & 7.44 & $.00^{* *}$ & .56 \\
\hline
\end{tabular}

Nota: (1) PAT=Plan de Acción Tutorial, SIT=Situación actual de la Tutoría=Tutoría, TIC= Tecnologías Información y la Comunicaci global $\odot$ Valoracincia Emocionalón. $(2)^{*}=p<.05 ;{ }^{* *}=p<.01 . M=$ Media, DT= Desviación Típica. (3) El tamaño del efecto estadístico está expresado con el valor $d$ de Cohen (1988): pequeño=.2; mediano $=.5$ y grande $=.8$.

\section{Estudio de regresión}

Para analizar qué factores son los que más predicen la satisfacción global con la tutoría universitaria, se ha llevado a cabo un estudio de regresión lineal mediante el método de pasos sucesivos, considerando a cada una de las dimensiones del cuestionario empleado variables predictoras y como variable criterio la pregunta satisfacción global con la tutoría universitaria. Se realizan tres regresiones, la primera para el total de la muestra, la segunda diferenciada por géneros (hombres y mujeres) y en tercer lugar una regresión distinta para cada Universidad (UJA y UMAZA).

En el primero de los estudios de regresión (véase Tabla 5), se obtuvieron resultados significativos en el modelo 1, explicando el factor Plan de Acción Tutorial un 52.2\% de la varianza $($ Beta $=.72 ; p=.00)$. Al introducir en el modelo 2 de regresión el factor Situación Actual de la Tutoría, los dos factores explican el $54.5 \%$ de la varianza $\left(R^{2}=.545\right)$, siendo significativos para Plan de Acción Tutorial y Situación Actual de la Tutoría, excluyéndose el factor -TIC-, siendo ambos predictores positivos.

Tabla 5

Análisis de regresión lineal (pasos sucesivos), variable criterio: Valoración global

\begin{tabular}{rlcccc}
\hline Modelo & Variables predictoras & R2 & Beta & $t$ & $p$ \\
\hline \multirow{2}{*}{ Modelo 1 } & (Constante) & .522 & & -10.14 & $.00^{* *}$ \\
& Plan Acción Tutorial (PAT) & & .72 & 30.72 & $.00^{* *}$ \\
\hline
\end{tabular}




\begin{tabular}{rlcccc}
\hline Modelo & Variables predictoras & R2 & Beta & $t$ & $p$ \\
\hline \multirow{3}{*}{ Modelo 2 } & (Constante) & .545 & & -11.95 & $.00^{* *}$ \\
& Plan Acción Tutorial (PAT) & & .55 & 15.99 & $.00^{* *}$ \\
& Situación Actual (SIT) & & .23 & 6.77 & $.00^{* *}$ \\
\hline
\end{tabular}

Nota: (1) PAT=Plan de Acción Tutorial, SIT=Situación actual Tutoría, TIC=Tecnologías Información y la Comunicación. (2) Variables excluidas en el Modelo 1: SIT y TIC, variables excluidas en el Modelo 2: TIC. (3) ${ }^{* *}=p<.01$.

El mayor poder de predicción apreciado en el coeficiente estandarizado lo tiene el Plan de Acción Tutorial (Beta $=.55 ; p=.00)$, seguido de la Situación Actual de la Tutoría (Beta=.23; $p=.00)$, excluyendo del modelo a las -TIC- $(p<.01)$.

Tabla 6

Análisis de regresión lineal (pasos sucesivos), variable criterio: Valoración global, diferenciado por género

\begin{tabular}{clcccc}
\hline \multicolumn{1}{c}{ Mujeres } & \multicolumn{1}{c}{ Variables predictoras } & R2 & Beta & $t$ & $p$ \\
\hline \multirow{2}{*}{ Modelo 1 } & (Constante) & .532 & & -8.409 & $.00^{* *}$ \\
& Plan Acción Tutorial (PAT) & & .730 & 25.03 & $.00^{* *}$ \\
& (Constante) & .556 & & -9.759 & $.00^{* *}$ \\
Modelo 2 & Plan Acción Tutorial (PAT) & & .551 & 12.802 & $.00^{* *}$ \\
& Situación Actual (SIT) & & .228 & 5.517 & $.00^{* *}$ \\
\hline \multirow{2}{*}{ Hombres } & Variables predictoras & $R^{2}$ & Beta & $t$ & $p$ \\
\hline \multirow{2}{*}{ Modelo 1 } & (Constante) & .501 & & -5.661 & $.00^{* *}$ \\
& Plan Acción Tutorial (PAT) & & .709 & 17.731 & $.00^{* *}$ \\
\multirow{2}{*}{ Modelo 2 } & (Constante) & .523 & & -6.836 & $.00^{* *}$ \\
& Plan Acción Tutorial (PAT) & & .547 & 9.535 & $.00^{* *}$ \\
& Situación Actual (SIT) & & .222 & 3.876 & $.00^{* *}$ \\
\hline
\end{tabular}

Nota: (1) PAT=Plan de Acción Tutorial, SIT=Situación actual Tutoría, TIC=Tecnologías Información y la Comunicación. (2) Variables excluidas en el Modelo 1: SIT y TIC, variables excluidas en el Modelo 2: TIC, para los dos géneros. (3) ${ }^{* *}=p<.01$.

En el segundo estudio de regresión diferenciado por género (véase Tabla 6) se han obtenido resultados similares a los de todos los participantes, tanto para el modelo 1 (factor Plan de Acción Tutorial) como para el modelo 2 que introduce la dimensión Situación Actual de la Tutoría (SIT), en ambos géneros; es decir, los resultados diferenciados por género siguen la misma tendencia que los obtenidos para el total. Los dos factores introducidos en el modelo 2 explican el $55.6 \%$ en el caso de las mujeres y el 52.3\% en el caso de los hombres, excluyéndose el factor Tecnologías de la Información (TIC), siendo predictores positivos de la satisfacción con la tutoría universitaria, en los dos géneros. La mayor predicción está en el factor PAT, tanto para las mujeres (Beta $=.551, p=.00, t=12.802$ ) como para los hombres (Beta $=.547, p=.00, t=9.535$ ). 
Tabla 7

Análisis de regresión lineal (pasos sucesivos), variable criterio: Valoración global, diferenciado por Universidad

\begin{tabular}{clcccc}
\hline UJA & \multicolumn{1}{c}{ Variables predictoras } & R2 & Beta & $t$ & $p$ \\
\hline \multirow{2}{*}{ Modelo 1 } & (Constante) & .588 & & -9.787 & $.00^{* *}$ \\
& Plan Acción Tutorial (PAT) & & .768 & 21.492 & $.00^{* *}$ \\
& (Constante) & .602 & & -10.523 & $.00^{* *}$ \\
\multirow{2}{*}{ Modelo 2 } & Plan Acción Tutorial (PAT) & & .625 & 11.716 & $.00^{* *}$ \\
& Situación Actual (SIT) & & .189 & 3.543 & $.00^{* *}$ \\
\hline \multirow{2}{*}{ UMAZA } & Variables predictoras & $R^{2}$ & Beta & $t$ & $p$ \\
\hline \multirow{2}{*}{ Modelo 1 } & (Constante) & .372 & & -2.144 & $.032^{*}$ \\
& Plan Acción Tutorial (PAT) & & .611 & 17.926 & $.00^{* *}$ \\
\multirow{2}{*}{ Modelo 2 } & (Constante) & .388 & & -3.565 & $.00^{* *}$ \\
& Plan Acción Tutorial (PAT) & & .494 & 10.826 & $.00^{* *}$ \\
& Situación Actual (SIT) & & .174 & 3.807 & $.00^{* *}$ \\
\hline
\end{tabular}

Nota: (1) PAT=Plan de Acción Tutorial, SIT=Situación actual Tutoría, TIC= Tecnologías Información y la Comunicación. (2) Variables excluidas en el Modelo 1: SIT y TIC, variables excluidas en el Modelo 2: TIC, para las dos Universidades. $(3)^{*}=p<.05,{ }^{* *} p<.01$.

En la Tabla 7, se pueden apreciar los resultados obtenidos para el tercero de los estudios de regresión realizados, en el cual se diferenciaban los datos en función de la Universidad de los participantes, quedando excluido del modelo 2 el factor TIC, al igual que ocurría en el resto de las regresiones realizadas. En este caso si hay variaciones en los datos obtenidos en cada una de las universidades, ya que el modelo 2 en la Universidad de Jaén (UJA) explica el 60.2\% de la varianza $\left(R^{2}=.602\right)$, mientras que en la Universidad Agustín Maza (UMAZA) el porcentaje de varianza explicado por los dos factores (PAT y SIT) del modelo 2 se reduce al 38.8\% $\left(R^{2}=.388\right)$, siendo ambos predictores positivos de la satisfacción con la tutoría universitaria (valor de $t$ significativo $p<.01$ ).

Igual ocurre con los poderes de predicción de cada factor asociados al coeficiente estandarizado (Beta), aunque coinciden en el orden en las dos universidades, en el caso de la UJA el valor de Beta es mayor que en la UMAZA, tanto para el Plan de Acción Tutorial -PAT- $($ Bet $a=.625, p=.00$ vs Beta $=.494, p=.00)$, como para la Situación Actual de la Tutoría -SIT- $($ Bet $a=.189, p=.00$ vs Beta=.174, $p=.00)$, por lo que se evidencia una ligera mayor predicción en la valoración global en la UJA.

\section{Discusión y conclusiones}

Tal y como se ha puesto de manifiesto en este trabajo, no existen apenas estudios que valoren los factores que condicionan la tutoría en la universidad y, menos aún, que lo hagan comparando realidades tan distantes, pero a la vez tan similares, como pueden ser la española y la argentina, sujetas a factores históricos, de lengua y de colaboración mutuas a lo largo de los años. Los modelos educativos también son aná- 
logos, por lo que los objetivos planteados en el estudio pueden ofrecer luces sobre la satisfacción del alumnado, el impacto de variables como el género y la participación en el PAT, así como los factores que ayuden a determinar en qué aspectos la tutoría puede contribuir a la calidad educativa.

La investigación ha contado con un total de 866 alumnos y ha analizado las diferencias en la percepción de la tutoría de estudiantes universitarios con la finalidad de indagar en los factores que condicionan la tutoría en la universidad, siguiendo una perspectiva comparada entre el alumnado de primer año de carrera en la Universidad de Jaén -UJA- (España) y la Universidad Agustín Maza -UMAZA- (Argentina). Para ello se han planteado tres grandes objetivos. El primero de ellos perseguía conocer la valoración del alumnado participante en el estudio en las dimensiones consideradas en el instrumento. Se puede afirmar que existe una buena valoración de los aspectos relacionados con el PAT y la situación actual de la tutoría, siendo algo más baja en el uso de las TIC en la labor tutorial. Estudios similares (Salomón y Morales, 2015; Lledó et al., 2016) señalan resultados similares en relación con sus expectativas en la orientación recibida, si bien Gil-Albarova et al. (2013), matizan el valor que los estudiantes confieren al trato humano del tutor. Otros aspectos destacados son el adecuado número de estudiantes por tutor, la estimación positiva de la tutoría y el uso de las TIC en la coordinación entre compañeros tutelados. Se aprecia una valoración positiva del uso de las TIC, lo que constata la realidad de que los tutores se apoyan en ellas para desarrollar de una manera más eficaz su labor tutorial (Cabello y Antón, 2005; Marqués, 2001). En nuestro estudio, destaca de manera clara cómo el uso de las aplicaciones sincrónicas en la comunicación (tipo mensajería instantánea) son consideradas muy por encima de las diacrónicas como el foro. Se constata poca presencia de las TIC en investigaciones previas. En la de García (2008), basada más en la opinión de los profesores, se ve el foro como un recurso de gran utilidad. Por otro lado, llama la atención que se reconozca una baja participación en las reuniones tutoriales o que no haya una buena orientación sobre las convocatorias de movilidad.

En cuanto al segundo objetivo, en el que se valora la influencia de la variable género, universidad y participación en el PAT, los máximos contrastes se obtienen en el tipo de universidad, a la postre uno de los ejes centrales de la investigación. La UMAZA obtiene sus principales diferencias con respecto a la UJA en la valoración del PAT y situación de la tutoría, pero esta tendencia se invierte en el caso del uso de las TIC. Es preciso destacar dos cuestiones que nos resultan relevantes. Por una parte, destaca la apuesta firme de la UMAZA por las tutorías, tal y como se ve en la introducción del presente artículo. Esto conlleva un Sistema Tutorial de más de diez años de trayectoria que falta en la UJA, además de una coordinación basada en incentivos que igualmente no existe en la universidad estudiada. Por otro lado, el hecho de que en la UJA se haya apostado desde hace años por el Campus Virtual dentro del proyecto de la Consejería de Innovación, Ciencia y Empresa de la Junta de Andalucía denominado "Universidad Digital" le confiere entidad propia al proyecto. En él participan las 10 universidades públicas andaluzas y se ofrece una docencia completamente virtual y a distancia mediante soporte tecnológico en forma de plataformas de enseñanza virtual. Esto hace que todo el alumnado, desde los primeros momentos de la vida universitaria, esté habituado al uso de las TIC en 
todos los procesos. Lo que se pretende significar es que, a veces, son las apuestas institucionales las que condicionan la valoración de los usuarios, por encima de la acción de las personas. La participación en el PAT es un indicador claro en el impacto que la tutoría pueda tener en la formación de los estudiantes, pues cataliza procesos de planificación y coordinación que de otra forma quedarían reducidos a acciones puntuales. En tal sentido, el estudio muestra claras diferencias a favor de quienes participan en las iniciativas de la universidad, frente a los que sólo asisten a tutorías de forma puntual, llegando a la misma conclusión otros estudios similares (CalderónGuerrero, Arraiza, y López Álvarez, 2014; Ponce et al., 2017).

En referencia al último objetivo, los factores que más predicen la satisfacción global de la tutoría universitaria son el PAT y la situación de la tutoría, por este orden. Estudios como el llevado a cabo por Aparicio, Lledó, Martínez, Gonzálvez, y Vicent (2016) analizaron los niveles de satisfacción de los estudiantes en los programas de tutorías incluidos en el PAT, hallando una alta satisfacción de los mismos, entre otros, en la metodología impartida, temáticas abordadas y vías de comunicación; sin embargo, Salomón y Morales (2015) determinaron un porcentaje significativo de alumnado que pensaba que no se estaban resolviendo sus problemas académicos y de forma integral, en especial por un bajo compromiso tutor-alumno y falta de asesoría académica. En otro extremo se sitúan los estudios que muestran un desconocimiento del alumnado acerca de lo que supone el PAT, a pesar de estar instaurado en la institución y funcionando (Lledó et al., 2016).

Como conclusión final de nuestro estudio, se subraya el hecho de que todo el alumnado que ha participado en los PAT valora positivamente esta iniciativa de la universidad, considera que repercute de forma notable en su formación y hace lo propio de la tutoría como función del profesorado, aunque todavía existen algunos aspectos que se deberían mejorar. La universidad UMAZA obtiene mejores resultados en los aspectos vinculados directamente con el diseño y puesta en marcha del PAT y de la tutoría, mientras que en la UJA destaca el uso de las TIC con fines orientadores.

Como principal limitación se destaca el hecho de no haber podido realizar una selección muestral, incluyendo al mismo alumnado con y sin participación en el PAT.

\section{Referencias}

Aguilar-Parra, J.M. et al (2015). Necesidades de formación del profesor universitario en competencias relacionadas con la acción tutorial. Revista de Docencia Universitaria, 13(3), 357-375. DOI: https://doi.org/10.4995/redu.2015.5433

Alonso-García, S., Rodríguez, A.M., y Cáceres, M.P. (2018). Análisis de la Acción Tutorial y su Incidencia en el Desarrollo Integral del Alumnado. El Caso de la Universidad de Castilla La Mancha (España). Formación universitaria, 11(3), 63-72. DOI: http:// dx.doi.org/10.4067/S0718-50062018000300063

Álvarez, P.R. (Coord.) (2012). Tutoría universitaria inclusiva. Madrid: Narcea.

Álvarez, P.R. (2013). La tutoría como eje articulador del proceso de aprendizaje del alumnado. Qurriculum: Revista de Teoría, Investigación y Práctica Educativa, 26, 73-87.

Aparicio, M.P., Lledó, A., Martínez, M.C., Gonzálvez, C. y Vicent, M. (2016). Plan de Acción Tutorial en la Facultad de Educación: satisfacción y propuestas de mejora 
del alumnado. En M.T. Tortosa-Ybáñez [Comps.], XIV Jornadas de Redes de Investigación en Docencia Universitaria (pp. 1905-1918). Alicante: Instituto de Ciencias de la Educación.

Arrebola, F., Morales, M.E., López, M., Martínez, M.A., Fernández, M., Rivas, A., Arias, J.L., Fernández, E. y Aguilera, M. (2016). Interdisciplinary collaboration experience: towards an effective tutorization of students in higher education. En L. Chova, [Eds.], Inted2016: 10th International Conference technology, education and development (pp. 1901-1906). Valencia: AITED. DOI: http://dx.doi.org/10.21125/inted.2016

Brical, J.M. (2000). Informe Universidad 2000. Recuperado de http://www.observatoriuniversitari.org/es/bricall_jm-2000-informe-universidad-2000/

Cabello, J. y Antón, P. (2005). Conversaciones con el profesorado. Un estudio en cuatro universidades españolas sobre el espacio europeo y el uso de las TIC. Revista de Educación, 337, 149-167.

Cabrera, L., Bethencourt, J.T., Álvarez, P.R. y González, M. (2006). El problema del abandono de los estudios universitarios. Revista Electrónica de Investigación y Evaluación Educativa (RELIEVE), 12(2), 171-203. DOI: https://doi.org/10.7203/relieve.12.2.4226

Caldera, J. F. (2015). Actitudes de los estudiantes universitarios ante la tutoría. Diseño de una escala de medición. Revista de la educación superior, 44(173), 103-124. DOI: https://doi.org/10.1016/j.resu.2015.04.004

Calderón-Guerrero, C., Arraiza, M.P. y López Álvarez, J.V. (2014). The tutorial action program at the ETSI Montes/UPM in the ehea frame: 5 years of experiencie in Erasmus studentes programs. En L. Chova [Comps.], 6TH International Conference on Education and new learning technologies (pp. 5567-5577). Barcelona: IATED.

Cano, R. (2009). Tutoría universitaria y aprendizaje por competencias. ¿Cómo lograrlo? Revista Electrónica Interuniversitaria de Formación del Profesorado, 12(1), 181-204.

Capelari, M. (2014). Las políticas de tutoría en la educación superior: génesis, trayectorias e impactos en Argentina y México. Revista Latinoamericana de Educación Comparada, 5(5), 41-54.

Cohen, J. (1988). Statistical Power Analysis for the Behavioral Sciences. Second Edition. Hillsdate, NJ: LEA.

Colvin, J., \& Ashman, M. (2010). Roles, Risks, and Benefits of Peer Mentoring Relationships in Higher Education. Mentoring \& Tutoring: Partnerership in Learning, 18(2), 121-134. DOI: https://doi.org/10.1080/13611261003678879

Da Re, L., Clerici, R. y Álvarez, P.R. (2017). The formative tutoring programme in preventing universit drop-outs and improving students' academic performance. The case stud of the Universit of Padova, (Italy). Journal of Sociology of Education, 9(3), 156-175. DOI: https://doi.org/10.14658/pupj-ijse-2017-3-7

De Backer, L. (2015). Promoting university students' metacognitive regulation through peer learning: the potential of reciprocal peer tutoring. Higher Education, 70(3), 469486. DOI: https://doi.org/10.1007/s10734-014-9849-3

De la Cruz, G. (2017). Tutoría en Educación Superior: análisis desde diferentes corrientes psicológicas e implicaciones prácticas. CPU-e Revista de Investigación Educativa, 25, 34-59.

De-Juan-Vigaray, M., \& García, C. (2017). Information overload or lack of interest: does the tutorial action plan (TAP) really stand out among the many university 
services on offer? En L. Chova, [Eds.], 11th International Conference on Technology, Education and Development INTED (pp. 4513-4522). Valencia: IATED. DOI: https:// doi.org/10.21125/inted.2017.1068

Díaz, V. (2012). Ventajas e inconvenientes de la encuesta por internet. Revista de Sociología, 97(1), 193-223.

Donoso, S. (2010). Retención de estudiantes y éxito académico en la educación superior: análisis de buenas prácticas. Informe de investigación. Talca (Chile): Instituto de Investigación y Desarrollo Educacional.

Fernández, F., Arco, J.L., López, S. y Heilborn, U. (2011). Prevención del fracaso académico universitario mediante tutoría entre iguales. Revista Latinoamericana de Psicología, 43(1), 59-71.

Fox, A., Stevenson, L. y Connelly, P. (2010). Peer-mentoring undergraduate accounting students: The influence on approaches to learning and academic performance. Active Learning in Higher Education, 11(2), 145-156. DOI: https://doi. org/10.1177/1469787410365650

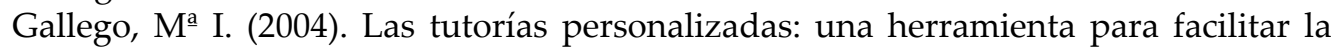
transición secundaria-universidad. En Actas XI Congreso Universitario de Innovación Educativa en las Enseñanzas Técnicas (pp. 65-75). Barcelona: UPC.

García, A. J., \& Troyano, Y. (2009). El Espacio Europeo de Educación Superior y la figura del profesor tutor en la Universidad. Revista de Docencia Universitaria, 7(2), 1-10. DOI: https://doi.org/10.4995/redu.2009.6244

García, A. (2008). La tutoría en la enseñanza universitaria y la contribución de las TIC para su mejora. Revista Electrónica de Investigación y Evaluación Educativa (RELIEVE), 14(2), 1-14.

Gaughf, Natalie, \& Foster, P. (2016). Implementing a centralized institutional peer tutoring program". Education for Health, 29(2), 148-151. DOI: https://doi.org/10.4103/13576283.188773

Gil-Albarova, A. (2013). Estudiantes universitarios y calidad del Plan de acción tutorial. Valoraciones y mejoras. Revista de Docencia Universitaria, 11(2), 63-87.

Giménez, J.A.; Cortés, M.T., y Motos, P. (2018). Cómo valoran los estudiantes los procesos de tutoría universitaria. En REDINE (Ed.), Innovative strategies for Higher for education in Spain (pp. 27-38). Eindhoven, N.L: Adaya Press.

Hagenauer, G., \& Volet, S. (2014). Teacher-student relationship at universit: an important yet under-researched field. Review of Education, 40(3), 370-388. DOI: https://doi. org/10.1080/03054985.2014.921613

Hall, R., \& Jaugietis, Z. (2011). Developing peer mentoring through evaluation. Innovative Higher Education, 36(1), 41-52. DOI: https://doi.org/10.1007/s10755-010-9156-6

Larose, S., Cyrenne, D., Garceau, O., Harvey, M., Guay, F., Godin, F., Tarabulsy, G.M., y Deschênes, C. (2011). Academic mentoring and dropout prevention for students in math, science and technology. Mentoring $\mathcal{E}$ Tutoring: Partnership in Learning, 19(4), 419-439. DOI: http://dx.doi.org/10.1080/13611267.2011.622078

Lim, H. (2002). Learner experience and achievement Project (LEAP). Survey Report 2002. Southampton Institute.

Lledó, A. et al. (2016). REDPAT-Educación: acompañamiento tutorial en la formación universitaria. En J. D. Álvarez [Comps.], Innovaciones metodológicas en docencia 
universitaria: resultados de investigación (pp. 2353-2361). Alicante: Universidad de Alicante, Vicerrectorado de Estudios, Formación y Calidad, Instituto de Ciencias de la Educación (ICE).

López, E. (2017). El concepto y las finalidades de la tutoría universitaria: una consulta a expertos, Revista Española de Orientación Psicopedagogía, 28(2), 61-78. DOI: https:// doi.org/10.5944/reop.vol.28.num.2.2017.20119

Lorenzo-Lledó, G., Lledó, A., Lorenzo, A. y Arraez, G. (2017). La acción tutorial como acompañamiento en el alumnado universitario con discapacidad: hacia una educación inclusiva. International Journal of Developmental and Educational Psychology, 138(1), 137-144. DOI: https://doi.org/10.17060/ijodaep.2017.n1.v4.1036

Martínez, M., \& Crespo, E. (2009). La tutoría universitaria en el modelo de la Convergencia Europea. En C. Gómez y S. Grau (Comps.), Propuestas de diseño, desarrollo e innovaciones curriculares y metodología en el EEES (pp. 451-466). Alcoy: Editorial Marfil.

Martínez, F., \& González, J. (2015). Uso y apropiación de las Tecnologías de la Información y la Comunicación por parte de los docentes en las facultades de ingeniería. Redes de Ingeniería, 6(1), 6-24. DOI: http://dx.doi.org/10.14483/udistrital.jour. redes.2015.1.a01

Marqués, P. (2001). Algunas notas sobre el impacto de las TIC en la universidad. Educar, 28, 83-98. DOI: http://dx.doi.org/10.5565/rev/educar.391

McKeegan, P. (1998). Using undergraduate teaching assistant in a research methodology course. Teaching in Psychology, 25(1), 11-14. DOI: https://doi.org/10.1207/ s15328023top2501_4

Muñoz, J.L., \& y Gairín, J. (2014). Orientación y tutoría durante los estudios universitarios: El plan de acción tutorial. Fuentes: Revista de la Facultad de Ciencias de la Educación, 14, 172-192.

Ocel, J. (2003). Outcomes of the gross and developmental anatomy teaching assistant experience. Clinical Anatomy, 16(6), 526-530. DOI: https://doi.org/10.1002/ca.10193

Pantoja, A. (2009). La acción tutorial ante el reto de la Tecnologías de la Información y la Comunicación (TIC). Madrid: Wolters Kluwer.

Ponce, L. (2017). Evaluación de la acción tutorial en la facultad de contaduría pública. Revista Electrónica sobre Educación Media y Superior, 4(7), 1-17.

Real Decreto 1791/2010, de 30 de diciembre, por el que se aprueba el Estatuto del Estudiante Universitario. BOE, 31 de diciembre de 2010. Recuperado de https:// www.boe.es/eli/es/rd/2010/12/30/1791

Salomón, J., \& Morales, M. H. (2015). Fortalezas, debilidades y satisfacción del programa de tutoría según estudiantes de medicina. Revista Iberoamericana de Producción Académica y Gestión Educativa, 2(3), 1-12.

Santos, F. et al. (2012). La Hora "TutHora". Revista del Congrés Internacional de Docència Universitària i Innovació (CIDUI), 1(1).

Schalk, K. (2009). The undergraduate teaching experience offers opportunities similar to the undergraduate research experience. Journal of Microbiology \& Biology Education, 10(1), 32-42.

Solaguren, M. y Moreno, L. (2019). Las tutorías académicas en carreras de ingeniería: una visión actual. Revista de Investigación Educativa, 37(1), 251-267. 
Strayhorn, T. (2012). College students' sense of belonging: A key to educational success for all students. New York: Routledge.

Tinto, V. (1975). Dropout from higher education: a theoretical synthesis of recent research. Review of Educational Research, 45(1), 89-125. DOI: https://doi. org/10.3102/00346543045001089

Torrado, M. (2004). Estudio de encuesta. En R. Bisquerra [Comp.], Metodología de la investigación educativa (pp. 231-257). Madrid: La Muralla.

Watts, T. (2011). Supporting undergraduate nursing students through structured personal tutoring: Some reflections. Nurse Education Today, 31(2), 214-218. DOI: https:// doi.org/10.1016/j.nedt.2010.06.005

Fecha de recepción: 24 de abril de 2019.

Fecha de revisión: 6 de mayo de 2019.

Fecha de aceptación: 4 de julio de 2019. 